\title{
Effects of Milsana and Benzothiadiazole on the Ultrastructure of Powdery Mildew Haustoria on Cucumber
}

\author{
Kirstin Wurms, Caroline Labbé, Nicole Benhamou, and Richard R. Bélanger
}

First, second, and fourth authors: Laboratoire de Biocontrôle, Département de Phytologie, Université Laval, Sainte-Foy, Québec, Canada G1K 7P4; and third author: Département de Phytologie, Recherche en Sciences de la Vie et de la Santé, Pavillon C. E. Marchand, Université Laval, Sainte-Foy, Québec, Canada G1K 7P4. Accepted for publication 17 May 1999.

\begin{abstract}
Wurms, K., Labbé, C., Benhamou, N., and Bélanger, R. R. 1999. Effects of Milsana and benzothiadiazole on the ultrastructure of powdery mildew haustoria on cucumber. Phytopathology 89:728-736.

Disease assessments and cytological investigations provided valuable information on the modes of action and efficacies of two prophylactic compounds, Milsana and benzothiadiazole (BTH), against powdery mildew development on long English cucumber. Milsana application significantly reduced disease incidence relative to inoculated controls through induction of localized resistance. Microscopic observations showed most haustoria had collapsed in the localized Milsana treatment and were encapsulated by an amorphous material impregnated by electron-opaque substances. The rapidity of haustorial collapse (within 4 days of treatment application) together with the encasement by electron-dense substances stained

blue by toluidine blue $\mathrm{O}$ suggest that phenolics are possibly involved in the Milsana defense response. Cytochemical labeling of chitin with a wheat germ agglutinin/ovomucoid-gold complex showed that complete cellular disorganization of the fungus had occurred without disturbance to chitin in the walls of mycelia and haustoria. This may indicate that chitinolytic activity is not important in the Milsana-activated defense response. Application of high doses of BTH induced occasional cell wall thickening and accumulation of a compound that stained purple by toluidine blue $\mathrm{O}$, but the defense response was weak, sporadic, and insufficient to reduce powdery mildew infection on cucumber. Responses to BTH could not be differentiated in terms of timing of the initial application or systemicity.

Additional keywords: Cucumis sativus, induced resistance, Sphaerotheca fuliginea.
\end{abstract}

Powdery mildew, caused by Sphaerotheca fuliginea (Schlechtend.:Fr.) Pollacci, is one of the major diseases affecting field and glasshouse cucumber production around the world $(3,32)$. Leaf infestation by this pathogen interferes with photosynthesis and respiration, leading to reduced fruit set, inadequate ripening, and poor flavor development (26). Disease control relies mainly on fungicides, but the combination of high inoculum pressure, glasshouse conditions that favor fungal growth, and exacting consumer standards for unblemished produce necessitates frequent pesticide applications. Consequently, control efficacy has been reduced by the emergence of resistant pathogen strains (27). Concerns about the environmental effects of fungicides have also led to increased restriction of their use, and as a result, other forms of control are urgently being sought. A promising approach in this regard is the use of prophylactic compounds such as benzothiadiazole (BTH) and Milsana (KHH BioSci Inc., Raleigh, NC).

$\mathrm{BTH}$, a novel chemical activator of plant disease resistance (16), has no known direct antifungal effect and is thought to play a role similar to that of salicylic acid in the signal transduction pathway that leads to systemic acquired resistance (SAR) (24). A single application to wheat provided protection against powdery mildew caused by the pathogen Blumeria graminis f. sp. tritici for an entire growing season, and yields were similar to those of plants treated with standard fungicides (16). The authors speculated that control was achieved through increased papilla formation and hypersensitive cell responses in the plant. However, in an earlier study, Aist (1) suggested that papilla formation alone was probably insufficient to prevent powdery mildew infection and the exact compounds involved in the hypersensitive response were not identified.

Corresponding author: R. R. Bélanger

E-mail address: richard.belanger@plg.ulaval.ca

Publication no. P-1999-0706-01R

(C) 1999 The American Phytopathological Society
Milsana is the registered name given to an extract from leaves of the giant knotweed, Reynoutria sachalinensis. Initially commercialized in 1990 as a wettable powder by Compo GmbH (Münster, Germany), an aqueous formulation (Milsana flüssig) was quickly developed because of the time-consuming nature of the original extraction procedure. The formulation was further improved in 1998 (KHH BioSci Inc.), but to date, there is no published data on the efficacy of the new product. Experiments with Milsana flüssig have shown that fruit yield was not adversely affected, control of powdery mildew on cucumber was comparable to that of the fungicide benomyl, and the mode of action appeared to involve the induction of plant defense responses rather than direct fungistasis (10). Peroxidases, $\beta-1,3-$ glucanases (20), and particularly phenolics (11) were among the defense molecules found to be enhanced by this product. In spite of such advances in our knowledge of how Milsana operates, little is known about the exact mechanisms underlying the expression of resistance and whether plant defense responses are localized or systemic. In this context, an ultrastructural investigation of the cellular events associated with Milsana-induced resistance appeared essential.

To address some of these issues, the objectives of the current study were to (i) compare the efficacy of BTH and the new formulation of Milsana at controlling cucumber powdery mildew, (ii) investigate the inducing effects of BTH and Milsana on host defense responses and fungal haustorium development, and (iii) assess the systemicity of resistance. In this paper, we provide the first evidence that Milsana-induced resistance is localized and results in the collapse of powdery mildew mycelia and haustoria, while high concentrations of BTH did not induce resistance against $S$. fuliginea on cucumber.

\section{MATERIALS AND METHODS}

Chemicals. American Chemical Society assurance chemicals were obtained from Canemco Inc. (Lachine, Québec, Canada), Commercial Alcohols (Brampton, Ontario, Canada), and Aldrich Chemical Company Inc. (Milwaukee, IN). 
Plant material and pathogen inoculation. Seeds of cucumber (Cucumis sativus L.) cultivar Corona were sown in Promix (Premier Horticulture, Rivière-du-Loup, Québec, Canada) and watered daily until emergence, whereupon they were placed in a growth cabinet, maintained at $24 / 20^{\circ} \mathrm{C}$ (day/night) with a 16 -h photoperiod of approximately $145 \mu \mathrm{E} \mathrm{m} \mathrm{m}^{-2} \mathrm{~s}^{-1}$. Fertilization with a sterilized nutrient solution (8) started at the one- to two-true-leaf stage. Applications were made three times per week in combination with $24 / 20^{\circ} \mathrm{C}$ (day/night) temperatures to induce rapid growth and twice weekly with a $23 / 19^{\circ} \mathrm{C}$ (day/night) differential when slower growth was required. Plants were watered until run-off every second day.

Inoculation was done at the four- to six-true-leaf stage. Powdery mildew conidia were obtained from naturally infected glasshousegrown cucumber plants, the leaves of which were shaken briskly $24 \mathrm{~h}$ before application to dislodge old, nonviable spores. Spores from a detached source leaf were tapped onto the adaxial surface of the first true leaf of each experimental plant that was to be inoculated. Spores were applied in the same manner to petri dishes with a known surface area $\left(60.8 \mathrm{~cm}^{2}\right)$ and volume $(20 \mathrm{ml})$ of sterile $1.5 \%$ (wt/vol) peptone water containing $0.01 \%$ (vol/vol) Tween 80 . Following 10 replicate counts of the petri dishes using a Hausser Scientific hemacytometer (VWR Scientific, Ville Mont-Royal, Québec, Canada), it was possible to ascertain that the inoculation process applied $4 \times 10^{3} \pm 9 \times 10^{2}$ spores per $\mathrm{cm}^{2}$ (mean \pm standard error). In addition to the inoculation of plants described above, inoculation of a further six plants was delayed until 5 days after application of BTH. Six plants were also maintained as uninoculated controls and were placed in a separate growth cabinet to prevent cross-contamination.

Treatment application, assessment of disease severity, and statistical analysis. Disease severity was assessed using percent area infection diagrams given by Spencer (37). Upon appearance of the first signs of infection, plants were randomly assigned to groups of six replicates and were sprayed to run-off (approximately $5 \mathrm{ml}$ per leaf) with (i) water ("inoculated control"); (ii) $2.8 \mathrm{mM}$ BTH ("BTH application after infection") $(4,16)$; or (iii) $0.5 \%$ (wt/vol) Milsana. The two final treatments comprised (iv) "uninoculated control," plants of which were sprayed with water; and (v) "BTH application before infection," in which BTH was applied 5 days prior to inoculation.

To examine local versus systemic responses, treatments were applied weekly to all leaves of each plant, except for true leaf 4 and leaf 9 , which were bagged during spraying.

Disease evaluations were performed twice weekly. Treatment effects on powdery mildew severity were assessed by analysis of variance on the nested, fixed effects design with means separation by $t$ tests of paired LSMeans $(P<0.05)$ using SAS software, version 6.12 TSO 40 (SAS Institute, Cary, NC).

TABLE 1. Assessment of the efficacy and systemicity of Milsana and benzothiadiazole (BTH) treatments against powdery mildew disease severity on old cucumber leaves

\begin{tabular}{lcccc}
\hline & \multicolumn{2}{c}{ Mean \pm SEM disease rating $^{\mathrm{a}}$} & & \\
\cline { 2 - 3 } Treatment & Local & Systemic $^{\mathrm{b}}$ & Probability $^{\mathrm{c}}$ & Significance \\
\hline Inoculated control & $2.83 \pm 0.44$ & $3.13 \pm 0.43$ & 0.7050 & n.s. $^{\mathrm{d}}$ \\
BTH before inoculation & $1.38 \pm 0.90$ & $1.83 \pm 0.48$ & 0.4830 & n.s. \\
BTH after inoculation & $2.17 \pm 0.17$ & $3.33 \pm 0.40$ & 0.1102 & n.s. \\
Milsana & $0.00 \pm 0.00$ & $3.50 \pm 0.22$ & 0.0001 & ***e
\end{tabular}

${ }^{a}$ Disease severity was rated according to percent leaf area infected, as described by Spencer (37). The assessment was carried out on 4 June 1998 (9 days after inoculation for the "BTH before inoculation" treatment and 17 days after inoculation for all other treatments). SEM = standard error of the mean.

${ }^{\mathrm{b}}$ Systemicity of the response was examined by comparing the local response on leaf 3 , which received direct treatment application, with the systemic response on leaf 4 , which was bagged during spraying.

${ }^{c}$ Probabilities refer only to comparisons between localized and systemic responses within each treatment, owing to the hierarchical nature of the nested design.

${ }^{\mathrm{d}}$ n.s. $=$ Nonsignificant $(P>0.05)$

e $* * *=$ Highly significant.
Tissue processing for light and transmission electron microscopy. Four days after treatment applications were made, fungal lesions (or uninfected leaf tissue in uninoculated controls) were sampled using a cork borer from both leaf 3 (local) and leaf 4 (systemic) of each of two plants per treatment. Samples were dissected into 1- $\mathrm{mm}^{2}$ pieces; fixed overnight at $4^{\circ} \mathrm{C}$ in $3 \%(\mathrm{vol} / \mathrm{vol})$ glutaraldehyde in $0.1 \mathrm{M}$ sodium cacodylate buffer, $\mathrm{pH} 7.2$; postfixed for $1 \mathrm{~h}$ at ambient temperature using $1 \%(\mathrm{wt} / \mathrm{vol})$ osmium tetroxide in the same buffer; dehydrated in an ethanol series; and infiltrated in Jembed 812 resin (Canemco Inc.). Thick sections ( 1 to $2 \mu \mathrm{m}$ ) were cut with glass knives for light microscopy and stained for $45 \mathrm{~s}$ with $0.2 \%$ (wt/vol) toluidine blue $\mathrm{O}$ in $2 \%$ (wt/vol) sodium borate, and 6 to 10 blocks per treatment were then examined for cells containing haustoria. Thin sections $(90 \mathrm{~nm})$ were cut with a diamond knife for transmission electron microscopy, mounted on nickel grids, stained with uranyl acetate and lead citrate, and examined with a JEOL 1200 EX transmission electron microscope (JEOL, Ltd., Tokyo) operating at $80 \mathrm{kV}$. Unless otherwise stated, reported observations were representative of all samples.

Cytochemical labeling and controls. Gold particles, averaging $12 \mathrm{~nm}$ in diameter, were prepared using the method of Frens (14). Wheat germ agglutinin (WGA), a lectin with $N$-acetylglucosamine binding specificity, was used in a two-step labeling procedure described previously (6) to visualize chitin. An ovomucoid-gold conjugate ( $\mathrm{pH}$ 6.0) was used as a second-step reagent. The specificity of the cytochemical probe was previously established by control tests (6). In these tests, labeling specificity was examined by (i) a substrate competition assay, whereby the ovomucoid-gold complex was incubated with WGA, to which was previously added an excess of $N-N^{\prime}-N^{\prime \prime}$-triacetylchitotriose (1 mg/ml of phosphate-buffered saline, $\mathrm{pH}$ 7.2); (ii) incubation with WGA, followed by unlabeled ovomucoid and finally by the ovomucoid-gold complex; and (iii) incubation with colloidal gold alone, to assess nonspecific adsorption of gold particles.

\section{RESULTS}

Assessment of disease severity. Direct application of Milsana to cucumber plants produced significant localized protection against powdery mildew (Fig. 1A and B) in both old (Table 1) and young (Table 2) leaves, but did not induce systemic protection. Differences in disease severity were visible within 4 days of treatment application. BTH was ineffective in controlling powdery mildew, regardless of whether it was applied before or after artificial inoculation, and localized and systemic responses could not be differentiated (Tables 1 and 2, Fig. 1C). In addition, prolonged use of $\mathrm{BTH}$ resulted in phytotoxic symptoms including leaf yellowing, while veins remained green (Fig. 1A).

TABLE 2. Assessment of the efficacy and systemicity of Milsana and benzothiadiazole (BTH) treatments against powdery mildew disease severity on young cucumber leaves

\begin{tabular}{lcccc}
\hline & \multicolumn{2}{c}{ Mean \pm SEM disease rating $^{\mathrm{a}}$} & & \\
\cline { 2 - 3 } Treatment & Local & Systemic $^{\mathrm{b}}$ & Probability $^{\mathrm{c}}$ & Significance \\
\hline Inoculated control & $3.30 \pm 0.37$ & $3.17 \pm 0.11$ & 0.8476 & n.s. $^{\mathrm{d}}$ \\
BTH before inoculation & $2.00 \pm 0.58$ & $1.70 \pm 0.77$ & 0.6657 & n.s. \\
BTH after inoculation & $2.20 \pm 0.92$ & $2.92 \pm 0.33$ & 0.3050 & n.s. \\
Milsana & $1.30 \pm 0.20$ & $3.17 \pm 0.17$ & 0.0102 & **e
\end{tabular}

a Disease severity was rated according to percent leaf area infected, as described by Spencer (37). The assessment was carried out on 8 June 1998 (13 days after inoculation for the "BTH before inoculation" treatment and 21 days after inoculation for all other treatments). SEM = standard error of the mean.

b Systemicity of the response was examined by comparing the local response on leaf 8 , which received direct treatment application, with the systemic response on leaf 9 , which was bagged during spraying.

c Probabilities refer only to comparisons between localized and systemic responses within each treatment, owing to the hierarchical nature of the nested design.

${ }^{\mathrm{d}}$ n.s. $=$ Nonsignificant $(P>0.05)$.

e $* *=$ Moderately significant. 
Light microscope observations. Light microscope observations reflected the treatment differences seen at the plant level (Fig. 2). Haustoria in the control treatment appeared well rounded and multilobed (Fig. 2B). In general, haustoria in the BTH treatments exhibited the same morphology (Fig. 2C, E, and F). However, an amorphous material surrounding the haustoria, which was stained purple by toluidine blue $\mathrm{O}$, was infrequently observed in BTH treat- ments (Fig. 2D). Accumulation of this material was not correlated to either timing of the initial BTH application relative to artificial inoculation (Fig. 2C and E versus D) or to systemicity (Fig. 2E and $\mathrm{F}$ versus $\mathrm{D}$ ). Moreover, this staining did not occur in samples collected after an extended period following inoculation. Haustoria were difficult to locate in the localized Milsana treatment, and approximately $80 \%$ of those that were observed exhibited pro-
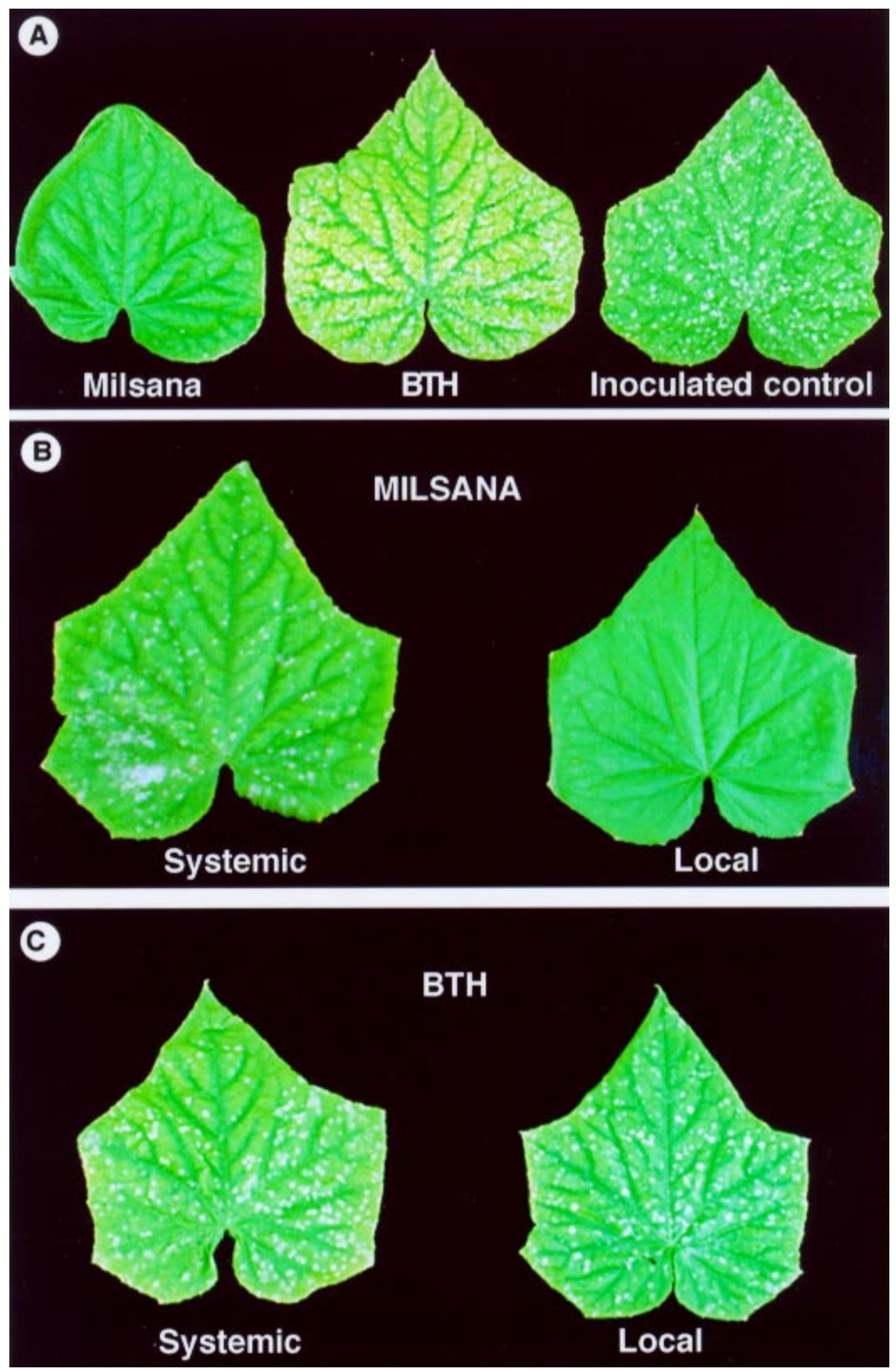

Fig. 1. Effect of Milsana and benzothiadiazole (BTH) on powdery mildew infection of long English cucumber plants. Photos were taken 18 days after the initial treatment application. A, Infection levels on leaves from treated plants versus inoculated controls. Loss of chlorophyll was evident in BTH-treated plants. B, Differences in infection obtained when Milsana was either applied directly (locally) to the leaves or leaves were shielded during spraying, allowing only for systemic transportation of any prophylactic responses. C, Localized and systemic responses to BTH. 

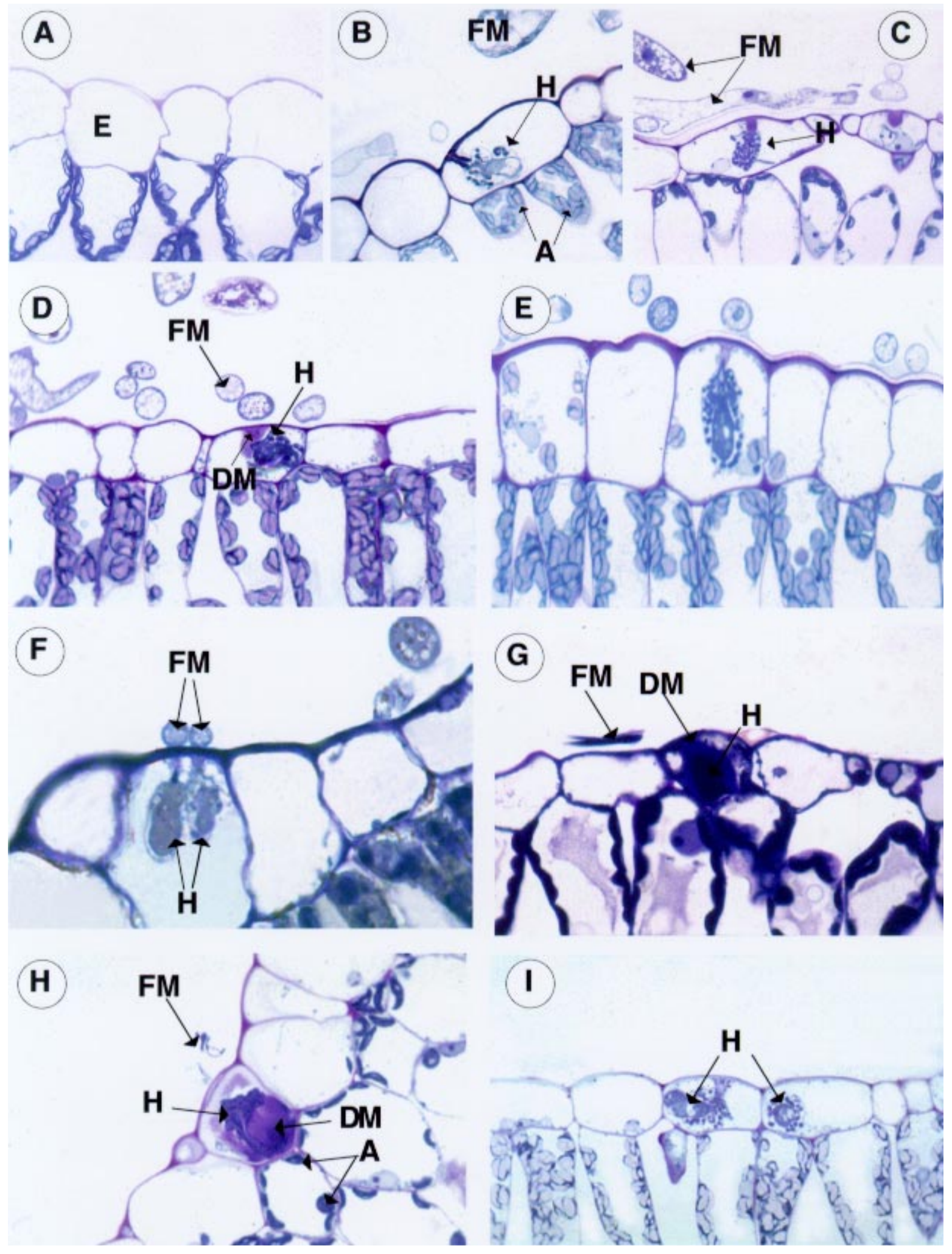

Fig. 2. Light micrographs of powdery mildew infections on cucumber leaves treated with $0.5 \%$ Milsana, $2.8 \mathrm{mM}$ benzothiadiazole (BTH), or water. Samples were collected 4 days after treatment application, and thick resin-embedded sections ( 1 to $2 \mu \mathrm{m}$ ) were stained with $0.2 \%$ toluidine blue $\mathrm{O}$ in $2 \%$ sodium borate. A, Normal epidermal and mesophyll cells in an uninoculated control. B, Powdery mildew in an inoculated control. C, Powdery mildew in a localized "BTH after inoculation" treatment. BTH was applied directly to the leaf 3 days after inoculation. D, Accumulation of dense material around a haustorium in a localized "BTH before inoculation" treatment. BTH was applied directly to the leaf tissue 5 days before inoculation with powdery mildew. E, Absence of dense material accumulation in localized "BTH before inoculation"-treated leaf tissue. F, Bipenetration in the systemic "BTH before inoculation" treatment. G, Dense material accumulation and haustorial collapse following localized Milsana application. H, Dense material accumulation and haustorial collapse in a localized Milsana treatment. I, Systemic response to Milsana. A = amyloplast, E = epidermal cell, $\mathrm{FM}=$ fungal mycelium, $\mathrm{H}=$ haustorium, and $\mathrm{DM}=$ dense material. 
nounced collapse (Fig. $2 \mathrm{G}$ and $\mathrm{H}$ ). This phenomenon was seldom observed in other treatments including BTH (Fig. 2B to F and I). In addition, collapsed haustoria in the localized Milsana treatment were encased by a dense material stained dark blue/purple by toluidine blue $\mathrm{O}$ (Fig. 2G and $\mathrm{H}$ ). In some cases, amyloplasts in plant mesophyll cells appeared smaller in the localized Milsana treatment relative to other treatments (Fig. 2B versus $\mathrm{H}$ ). No other treatment effects could be clearly distinguished, and in general, wellformed haustoria (Fig. 2B, C, E, F, and I) were abundant in leaves from all treatments except localized Milsana.

Transmission electron microscope observations. Examination of samples under transmission electron microscopy confirmed the light microscope observations and revealed additional ultrastructural details. S. fuliginea haustorium morphology, which predominated in all treatments except localized Milsana, was characterized by an oval central body, flanked by lobes (Fig. 3A to D), and surrounded by an extrahaustorial membrane derived from the host plasmalemma (7). Organelles such as mitochondria, embedded in ribosome-rich cytoplasm, were frequently observed within haustoria (Fig. 3C). The neck of each haustorium was surrounded by a multilayered collar. Two layers were often distinguishable in this collaran electron-lucent amorphous region immediately adjacent to the neck and an electron-dense granular/fibrillar area adjacent to the extrahaustorial membrane (Fig. 3A).

Haustoria observed in leaves treated with Milsana were markedly distorted as compared with those found in control plants. They had retracted completely from the extrahaustorial membrane (Figs. 3E and 4D), and collapsed lobes were lying directly around the main haustorial body (Fig. 4A). Such altered haustoria were electron dense, and cell organelles could not be distinguished (Figs. 3E and
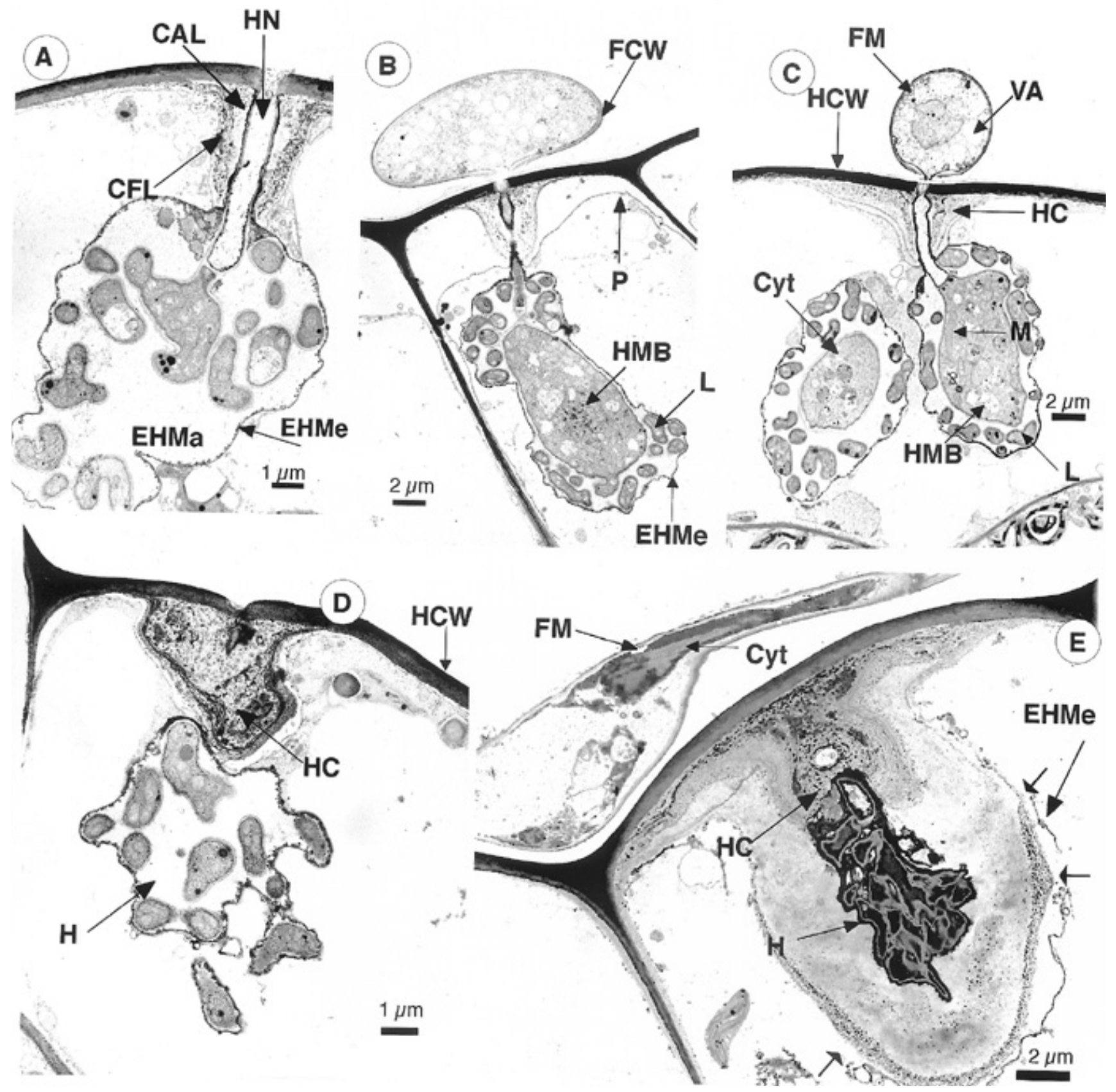

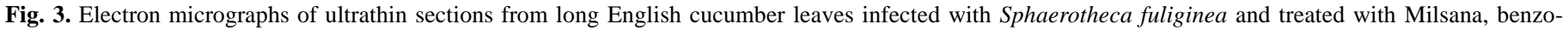

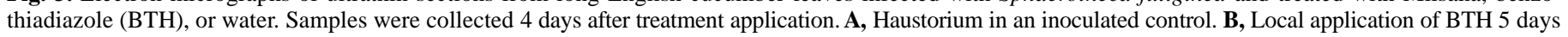

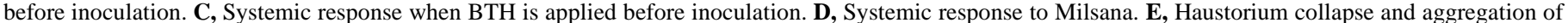

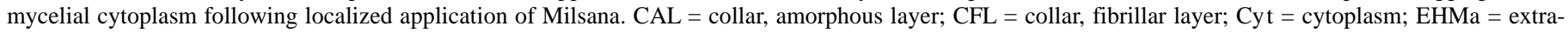

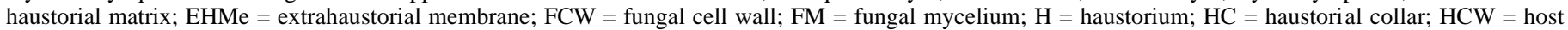
cell wall; $\mathrm{HMB}=$ haustorial main body; $\mathrm{HN}=$ haustorial neck; $\mathrm{L}=$ lobe; $\mathrm{M}=$ mitochondrion; $\mathrm{P}=$ plasmalemma; and $\mathrm{Va}=\mathrm{vacuole}$. 
4A). The space between the collapsed haustorium and the extrahaustorial membrane was filled with an amorphous material, the margin of which was delineated by an osmiophilic granular deposit, directly adjacent to the convoluted extrahaustorial membrane, which had ruptured in places (Fig. 3E, small arrows).

Cytoplasm aggregation, plasmalemma retraction, absence of organelles, and in some cases complete structural collapse were typical features of powdery mildew cells lying on the host surface in the localized Milsana treatment (Figs. 4B and 5C). Mycelia in all other treatments were typically electron-lucent, and organelles were readily distinguishable (Fig. 3C). In leaves sprayed directly with Milsana, the collar of the haustorium was more defined and there appeared to be two additional semigranular layers at the extremity of the collar that were impervious to the lead citrate-uranyl acetate stain (Fig. 4B). Multitextured deposits were sometimes present along the length of host cell walls adjacent to haustoria in both Milsana (Fig. 4C) and BTH treatments, but cell wall thickening was not consistently observed at all penetration sites. In contrast, accumulation of osmiophilic material in the region between the collapsed haustorium and the extrahaustorial membrane was a consistent feature of the host response in the localized Milsana treatment (Fig. 4D).
Cytochemical labeling. Only the mycelial cell wall and the haustorial wall were labeled by the WGA/ovomucoid-gold complex (Fig. 5). All plant structures and the extrahaustorial matrix were chitin-free (Fig. 5A). This labeling pattern was the same regardless of treatment (Fig. 5A versus D and B versus C). Localized Milsana application had caused extensive internal disorganization to the fungal mycelium including cytoplasm aggregation and retraction of the plasmalemma from the cell wall, but this damage had occurred without disruption of the cell wall as evidenced by preservation of the chitin label (Fig. 5C). Lobes were lying closer together in the partially collapsed haustorium from the localized Milsana treatment, but even-labeling of the haustorial walls indicated that they were still intact (Fig. 5D).

\section{DISCUSSION}

Comparative investigations of the effects of Milsana and BTH on the powdery mildew-cucumber interaction indicated that only Milsana application induced localized resistance, resulting in aggregation and collapse of pathogen cells and accumulation of an electron-dense material around the penetrating structures. In contrast, BTH was an ineffectual compound under the experimental
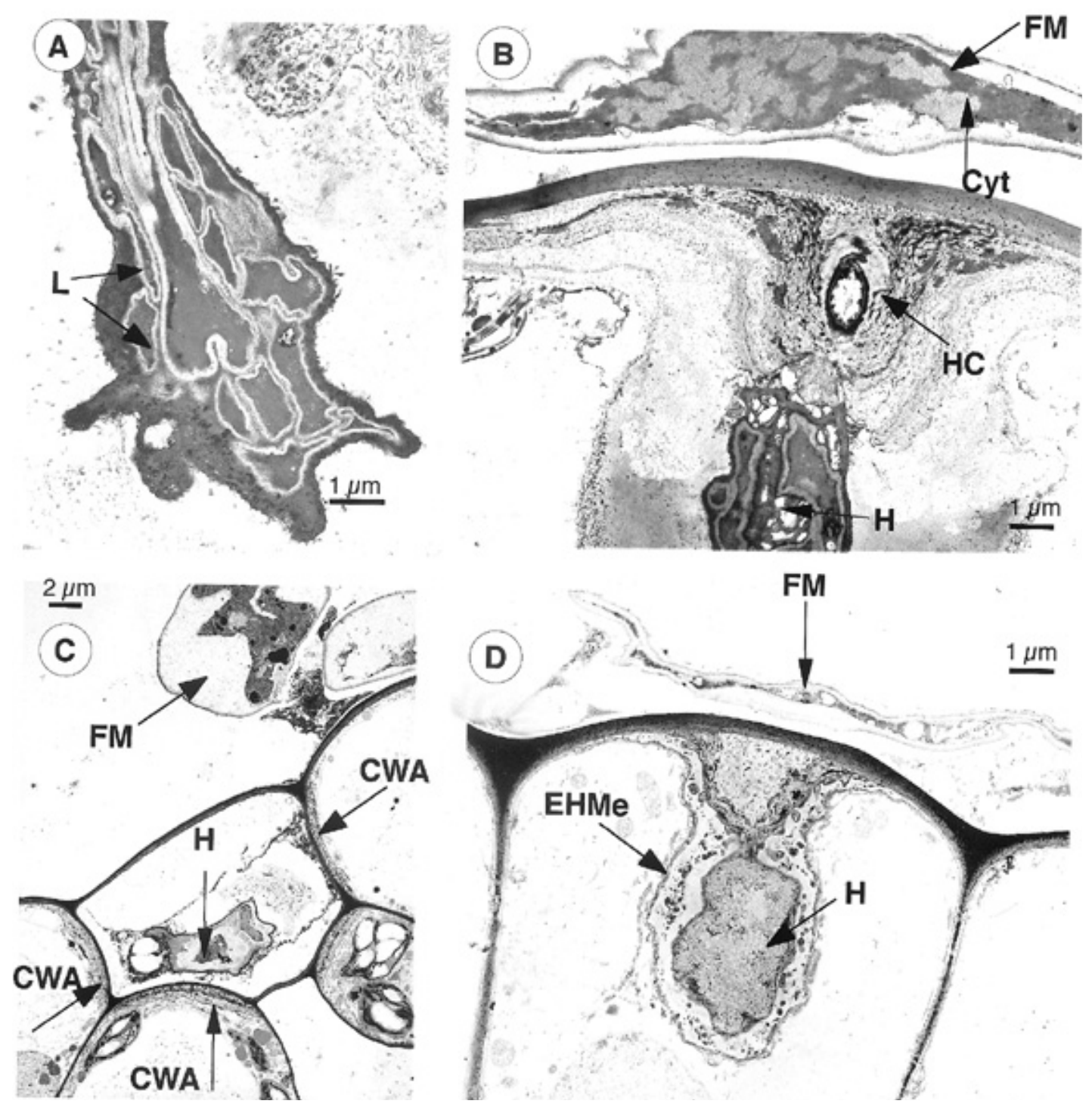

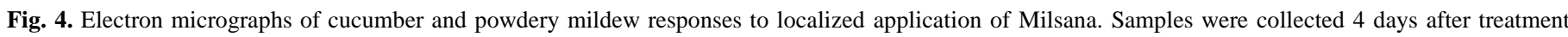
application. A, Close-up of a collapsed haustorium. B, Cytoplasm aggregation within a powdery mildew mycelium and thickening of the collar that surrounds the neck of the haustorium. C, Cell wall appositions surrounding an infected epidermal cell. D, Retraction of a haustorium from the extrahaustorial membrane. The haustorium is in the early stages of collapse. $\mathrm{CAL}=$ collar, amorphous layer; $\mathrm{CFL}=$ collar, fibrillar layer; $\mathrm{CWA}=$ cell wall apposition; $\mathrm{Cyt}=$ cytoplasm; $\mathrm{EHMe}=$ extrahaustorial membrane; $\mathrm{FM}=$ fungal mycelium; $\mathrm{H}=$ haustorium; and $\mathrm{L}=$ lobe. 

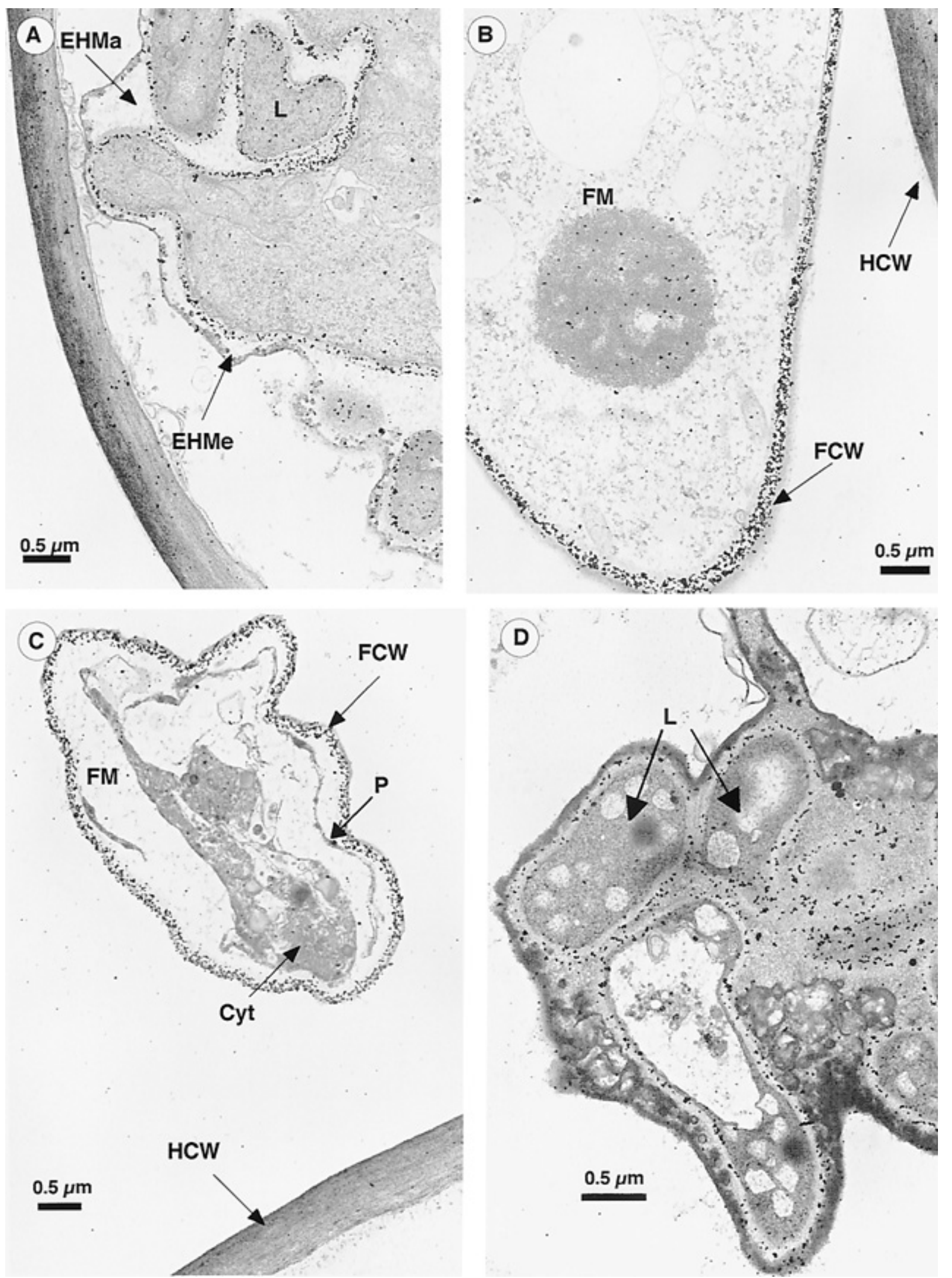

Fig. 5. Electron micrographs of chitin localization in cucumber leaves infected by powdery mildew and treated with water or Milsana. Samples were labeled with the wheat germ agglutinin/ovomucoid-gold complex. A, Chitin localization in an inoculated control sample showing the specific deposition on gold particles along the walls of haustorial lobes and the main haustorial body. This labeling pattern is representative of the response observed in all leaves treated with benzothiadiazole (BTH) and in the systemic response to Milsana (data not shown). B, Chitin localization within the wall of a powdery mildew mycelium in the inoculated control. C, Labeling of a mycelial cell wall following localized Milsana application. Plasmalemma retraction and cytoplasm aggregation have occurred without disturbance of chitin in the cell wall. D, Chitin labeling of a partially collapsed haustorium following localized Milsana application. CAL $=$ collar, amorphous layer; CFL $=$ collar, fibrillar layer; $\mathrm{Cyt}=$ cytoplasm; $\mathrm{EHMa}=$ extrahaustorial matrix; $\mathrm{EHMe}=$ extrahaustorial membrane; $\mathrm{FCW}=$ fungal cell wall; $\mathrm{FM}=$ fungal mycelium; $\mathrm{H}=$ haustorium; $\mathrm{HC}=$ haustorial collar; $\mathrm{HCW}=$ host cell wall; $\mathrm{HMB}=$ haustorial main body; $\mathrm{HN}=$ haustorial neck; $\mathrm{L}=$ lobe; $\mathrm{M}=$ mitochondrion; $\mathrm{P}=$ plasmalemma; and $\mathrm{Va}=$ vacuole. 
conditions used in this particular system. Milsana significantly reduced disease incidence on the plant and induced obvious ultrastructural damage to fungal cells and haustoria, as evidenced by cytoplasm aggregation, loss of cell organelles, and tissue collapse. In contrast, the proliferation of ribosomes, organelles, and cell division in most fungal structures examined in all of the other treatments was indicative of a highly active metabolism $(7,25)$. Our observations of haustoria formed in control plants also correlate well with descriptions in other reports $(17,33)$. Given that Milsana is not inherently fungitoxic $(10,11)$, pathogen degradation is most likely associated with plant defense responses. Induction of host defense reactions is an energy-demanding process (35) and may explain the reduced size of amyloplasts in the localized Milsana treatment due to depletion of starch reserves.

Although chitinolytic activity and lignification have often been reported to be major components of general cucumber resistance $(18,19,21,29)$, the results from this study tend to indicate that chitinases are not major components in Milsana-mediated induced resistance of cucumber against powdery mildew. Both Milsana and BTH are known to induce chitinase activity in cucumber $(22,34)$, but analysis of the labeling pattern obtained with the WGA/ovomucoidgold complex in our study showed that fungal cytoplasm in the localized Milsana treatment underwent pronounced disorganization without disturbance of chitin in the cell walls. In addition, chitin localization patterns in the inoculated control, BTH, and Milsana samples were indistinguishable. These observations indicate that fungal cell walls have not been decomposed in spite of chitinase induction, suggesting that enzyme-mediated wall hydrolysis is not an integral component of the Milsana-induced defense reaction. Elicitation of a plant defense response does not in itself constitute evidence of an efficacious disease control mechanism; for instance, chitinase elicitation in cucumber could not be correlated with resistance to several pathogens $(12,30)$. However, this research shows that cellular localization can be a useful tool for providing additional indirect evidence of the relative importance of a defense reaction.

Papilla formation is another reported defense mechanism that did not appear to play a key role in the localized Milsana defense response. It has been suggested that the collar-like structures that frequently surround the necks of haustoria originate from papillae (25) and that their presence is usually associated with poor haustorial development (17). However, the very presence of haustoria suggests that they have already successfully penetrated the papillate mechanical barrier, and in our experiment, collars were present in equal abundance in all treatments, with no apparent adverse effects on haustorial health.

Cell wall appositions were observed on occasion (Fig. 4C) and are thought to adversely affect the pathogen by restricting water and metabolite diffusion from the host into haustoria (25). However, the sporadic occurrence of these appositions suggests that their role in resistance within this system may be limited.

Several lines of evidence lead us to suggest that phenolics may be involved in the Milsana defense response, although position confirmation requires use of a specific probe. Localized application of Milsana resulted in rapid (within 4 days of application) and extensive collapse of fungal structures and the encasement of haustoria in dense materials. This collapse could have resulted either from the formation of an impenetrable barrier around the haustorium, preventing the flow of water and nutrients and causing a slow starvation, or from the direct interaction of fungal structures with toxic compounds such as phytoalexins. Given the speed of the response, the latter explanation appears more likely. Although the toluidine blue $\mathrm{O}$ reaction cannot be considered highly specific, it is known to stain polyphenolic compounds such as lignin a bluegreen color $(4,36)$. The intense blue coloration observed around collapsed haustoria in the localized Milsana treatment may well correspond to phenolic accumulation. Moreover, the granular electron-dense deposits observed adjacent to the extrahaustorial mem- brane are typically associated with phenolic compounds (9). Unlike many other defense mechanisms that may be induced systemically, phenolic accumulation is typically localized $(2,23,31)$, as was the resistance induced by Milsana. The work of Daayf et al. (11) has shown that several phenolic compounds with antifungal activity were elicited by Milsana flüssig and that kinetics of accumulation of one of these compounds correlated well with the onset of resistance. Indeed, phytoalexins may be an integral part of a generalized cucumber defense response against powdery mildew, given that they are an important factor of resistance mediated by other inducers such as silicon $(13,28)$.

While previous studies $(4,16)$, albeit in different systems, have reported the prophylactic role of BTH through induction of defense responses, our results did not confirm such a role in the cucumber-S. fuliginea infection. However, elicitor concentration and timing of induction need to be optimized before it can be concluded that BTH is ineffective in this pathosystem. Although the concentration of BTH used in this study was high (2 to 10 times that routinely used in other studies) $(15,16)$ and prolonged use of this concentration appeared to cause phytotoxicity symptoms, inefficacy of the product was not related to phytotoxicity. BTH concentrations of 1.4 to $3.2 \mathrm{mM}$ have been shown to effectively induce resistance without any apparent phytotoxicity in wheat, tomato, and cucumber $(4,5,16)$. In addition, these concentrations do not appear to have any toxic effects on a range of fungal pathogens $(4,15)$. Elicitor treatments were applied weekly in our study to induce defense mechanisms against new generations of powdery mildew that are generated on a continual basis by the polycyclic fungus. Phytotoxic symptoms only became evident in the third week of the experiment (data not shown), following repeat applications of the elicitor, but BTH was an ineffective protectant throughout the trial, irrespective of the presence or absence of phytotoxicity. In addition, phytotoxic damage was not severe, since the obligate parasite was able to thrive in symptomatic tissue, and the plant was still capable of initiating defenses such as sporadic cell wall thickening and papilla formation.

Although our study evaluated two different times of initial BTH application, the optimal period required for induction of defense mechanisms and the duration of the defense response remain to be established for this system. The initial application of BTH was made both in advance of and after artificial inoculation, so that results would be respectively comparable with protocols used in other BTH trials $(4,5,16)$ and with the timing of the initial Milsana application in our study. When the first application of BTH was made after artificial inoculation, only primary colonies of powdery mildew were present on leaf 1 , but disease severity was evaluated on colonies on older leaves that developed subsequent to BTH application. Consequently, the prophylactic capacity of BTH was always investigated, regardless of the timing of the initial elicitor application, since BTH is not known to be curative (16). To determine the optimal timing for induction, a larger range of initial application times would need to be tested.

Other possible explanations for the ineffectiveness of BTH in this study are (i) the degree of the defense response was insufficient to restrict the pathogen, or (ii) the types of mechanisms induced were ineffective against the pathogen. Although there was some activation of host defense responses, as evidenced by sporadic wall thickening and occasional accumulation of a purplestained compound around some haustoria, these defense reactions were clearly not sufficient to reduce disease or to cause the level of fungal cell damage that was observed in the Milsana treatment. The specific defense mechanisms stimulated by BTH in the cucumber-powdery mildew system were not established in our study. In other studies, BTH has been shown to activate SAR in several plants including Arabidopsis, cucumber, tobacco, and wheat $(4,15$, 16,24). BTH-mediated SAR is normally associated with the synthesis of pathogenesis-related (PR) proteins including those from the PR-1, -2, -3, and -5 families $(15,16,22,24)$, while induced syn- 
thesis of secondary metabolites has only been reported on one occasion (4). PR proteins such as hydrolytic enzymes can be effective mechanisms in other systems, but if elicited, were not an effective component of the cucumber defense response against powdery mildew. Hence, the limited degree of the defense response together with the types of mechanisms activated may, in part, explain why BTH was ineffective at controlling powdery mildew on cucumber.

In conclusion, this study has provided data on the efficacy of the new Milsana formulation and the first in situ evidence of its localized mode of action. Pronounced collapse of fungal structures was accompanied by accumulation of electron-dense and amorphous materials, and the possibility that these compounds are phenolics deserves further investigation. BTH has shown great promise as a prophylactic compound in other host-pathogen systems but did not reduce disease or produce any significant ultrastructural responses in the cucumber-S. fuliginea interaction of this study.

\section{ACKNOWLEDGMENTS}

This work was supported, in part, by the Natural Sciences and Engineering Research Council of Canada. We thank A. Goulet and D. Auclair for excellent technical assistance.

\section{LITERATURE CITED}

1. Aist, J. R. 1976. Papillae and related wound plugs of plant cells. Annu. Rev. Phytopathol. 14:145-163.

2. Anderson, A. J. 1991. Phytoalexins and plant resistance. Pages 569-594 in: Mycotoxins and Phytoalexins. R. P. Sharma and D. K. Salunkhe, eds. CRC Press, Boca Raton, FL.

3. Askary, H., Benhamou, N., and Brodeur, J. 1997. Ultrastructural and cytochemical investigations of the antagonistic effect of Verticillium lecanii on cucumber powdery mildew. Phytopathology 87:359-368.

4. Benhamou, N., and Bélanger, R. R. 1998. Induction of systemic resistance to Pythium damping-off in cucumber plants by benzothiadiazole: Ultrastructure and cytochemistry of the host response. Plant J. 14:13-21.

5. Benhamou, N., and Bélanger, R. R. 1998. Benzothiadiazole-mediated induced resistance to Fusarium oxysporum f. sp. radicis-lycopersici in tomato. Plant Physiol. 118:1203-1212.

6. Benhamou, N., Rey, P., Chérif, M., Hockenhull, J., and Tirilly, Y. 1997. Treatment with the mycoparasite Pythium oligandrum triggers induction of defense-related reactions in tomato roots when challenged with $\mathrm{Fu}$ sarium oxysporum f. sp. radicis-lycopersici. Phytopathology 87:108-122.

7. Bushnell, W. R., and Gay, J. 1978. Accumulation of solutes in relation to the structure and function of haustoria in powdery mildews. Pages 183-235 in: The Powdery Mildews. D. M. Spencer, ed. Academic Press, London.

8. Chérif, M., Asselin, A., and Bélanger, R. R. 1994. Defense responses induced by soluble silicon in cucumber roots infected by Pythium spp. Phytopathology 84:236-242.

9. Chérif, M., Benhamou, N., and Bélanger, R. R. 1991. Ultrastructural and cytochemical studies of fungal development and host reactions in cucumber plants infected by Pythium ultimum. Physiol. Mol. Plant Pathol. 39:353-375.

10. Daayf, F., Schmitt, A., and Bélanger, R. R. 1995. The effects of plant extracts of Reynoutria sachalinensis on powdery mildew development and leaf physiology of long English cucumber. Plant Dis. 79:577-580.

11. Daayf, F., Schmitt, A., and Bélanger, R. R. 1997. Evidence of phytoalexins in cucumber leaves infected with powdery mildew following treatment with leaf extracts of Reynoutria sachalinensis. Plant Physiol. 113:719-727.

12. Dalisay, R. F., and Kuć, J. A. 1995. Persistence of reduced penetration by Colletotrichum lagenarium into cucumber leaves with induced systemic resistance and its relation to enhanced peroxidase and chitinase activities. Physiol. Mol. Plant Pathol. 47:329-338.

13. Fawe, A., Abou-Zaid, M., Menzies, J. G., and Bélanger, R. R. 1998. Silicon-mediated accumulation of flavonoid phytoalexins in cucumber. Phytopathology 88:396-401.

14. Frens, G. 1973. Controlled nucleation for regulation of the particle size in monodisperse gold suspensions. Nature (Lond.) Phys. Sci. 241:20-22.

15. Friedrich, L., Lawton, K., Ruess, W., Masner, P., Specker, N., Gut Rella,
M., Meier, B., Dincher, S., Staub, T., Uknes, S., Métraux, J.-P., Kessmann, H., and Ryals, J. 1996. A benzothiadiazole derivative induces systemic acquired resistance in tobacco. Plant J. 10:61-70.

16. Görlach, J., Volrath, S., Knauf-Beiter, G., Hengy, G., Beckhove, U., Kogel, K.-H., Oostendorp, S., Staub, T., Ward, E., Kessmann, H., and Ryals, J. 1996. Benzothiadiazole, a novel class of inducers of systemic acquired resistance, activates gene expression and disease resistance in wheat. Plant Cell 8:629-643.

17. Hajlaoui, M. R., Benhamou, B., and Bélanger, R. R. 1991. Cytochemical aspects of fungal penetration, haustorium formation and interfacial material in rose leaves infected by Sphaerotheca pannosa var. rosae. Physiol. Mol. Plant Pathol. 39:341-355.

18. Hammerschmidt, R., Bonnen, A. M., Bergstrom, G. C., and Baker, K. K. 1985. Association of epidermal lignification with non-host resistance of cucurbits to fungi. Can. J. Bot. 63:2393-2398.

19. Hammerschmidt, R., and Kuć, J. 1982. Lignification as a mechanism for induced systemic resistance in cucumber. Physiol. Plant Pathol. 20:60-71.

20. Herger, G., and Klingauf, F. 1990. Control of powdery mildew fungi with extracts of the giant knotweed, Reynoutria sachalinensis (Polygonaceae). Meded. Fac. Landbouwwet. Rijksuniv. Gent 55:1007-1014.

21. Irving, H. R., and Kuć, J. A. 1990. Local and systemic induction of peroxidase, chitinase and resistance in cucumber plants by $\mathrm{K}_{2} \mathrm{HPO}_{4}$. Physiol. Mol. Plant Pathol. 37:355-366.

22. Kastner, B., Tenhaken, R., and Kauss, H. 1998. Chitinase in cucumber hypocotyls is induced by germinating fungal spores and by fungal elicitor in synergism with inducers of acquired resistance. Plant J. 13:447-454.

23. Kuć, J. 1995. Phytoalexins, stress metabolism, and disease resistance in plants. Annu. Rev. Phytopathol. 33:275-297.

24. Lawton, K. A., Friedrich, L., Hunt, M., Wyemann, K., Delaney, T., Kessmann, H., Staub, T., and Ryals, J. 1996. Benzothiadiazole induces disease resistance in Arabidopsis by activation of the systemic acquired resistance signal transduction pathway. Plant J. 10:71-82.

25. Manners, J. M., and Gay, J. L. 1983. The host-parasite interface and nutrient transfer in biotrophic parasitism. Pages 163-195 in: Biochemical Plant Pathology. J. A. Callow, ed. John Wiley \& Sons Ltd., Chichester, United Kingdom.

26. McGrath, M. T. 1996. Successful management of powdery mildew in pumpkin with disease threshold-based fungicide programs. Plant Dis. 80: 910-916.

27. Menzies, J. G., and Bélanger, R. R. 1996. Recent advances in cultural management of diseases of greenhouse crops. Can. J. Plant Pathol. 18: 186-193.

28. Menzies, J. G., Ehret, D. L., Glass, A. D. M., and Samuels, A. L. 1991. The influence of silicon on cytological interactions between Sphaerotheca fuliginea and Cucumis sativus. Physiol. Mol. Plant Pathol. 39:403-414.

29. Métraux, J. P., and Boller, T. 1986. Local and systemic induction of chitinase in cucumber plants in response to viral, bacterial and fungal infections. Physiol. Mol. Plant Pathol. 28:161-169.

30. Nakajima, K., Kawaguchi, M., Kato, J., Oishi, K., Kanehira, T., and Shinohara, M. 1992. Enzymological and immunological properties of chitinases in Fusarium-infected cucumbers. Pages 408-418 in: Advances in Chitin and Chitosan. C. J. Brine, P. A. Sandford, and J. P. Zikakis, eds. Elsevier Science Publishers Ltd., London.

31. Nicholson, R. L., and Hammerschmidt, R. 1992. Phenolic compounds and their role in disease resistance. Annu. Rev. Phytopathol. 30:369-389.

32. Reuveni, M., Agapov, V., and Reuveni, R. 1996. Controlling powdery mildew caused by Sphaerotheca fuliginea in cucumber by foliar sprays of phosphate and potassium salts. Crop Prot. 15:49-53.

33. Samuels, A. L., Glass, A. D. M., Menzies, J. G., and Ehret, D. L. 1994. Silicon in cell walls and papillae of Cucumis sativus during infection by Sphaerotheca fuliginea. Physiol. Mol. Plant Pathol. 44:237-242.

34. Schneider, S., and Ullrich, W. R. 1994. Differential induction of resistance and enhanced enzyme activities in cucumber and tobacco caused by treatment with various abiotic and biotic inducers. Physiol. Mol. Plant Pathol. 45:291-304.

35. Smedegaard-Peterson, V., and Tolstrup, K. 1985. The limiting effect of disease resistance on yield. Annu. Rev. Phytopathol. 23:475-490.

36. Southern, S. G., and Deverall, B. J. 1990. Histochemical and chemical evidence for lignin accumulation during the expression of resistance to leaf rust fungi in wheat. Physiol. Mol. Plant Pathol. 36:483-494.

37. Spencer, D. M. 1977. Standardized methods for the evaluation of fungicides to control cucumber powdery mildew. Pages 455-464 in: Crop Protection Agents-Their Biological Evaluation. N. R. McFarlane, ed. Academic Press, London. 